\title{
Radiofrequency catheter ablation: a new frontier in interventional cardiology
}

\author{
Y Bashir, D E Ward
}

The development of non-pharmacological approaches for controlling cardiac arrhythmias reflects the many limitations of longterm antiarrhythmic drug therapy. These include poor patient compliance, frequent treatment failures or side effects or both, and a small but definite risk of life-threatening toxicity or proarrhythmia. Antiarrhythmic surgery has proved highly effective in many arrhythmias but it is expensive, causes considerable discomfort, and is associated with a definite incidence of morbidity and mortality. Curative ablation of arrhythmia substrates by catheter-based techniques avoids many of these problems and is far more acceptable to patients and physicians. In 1982, Gallagher and Scheinman separately described successful closed chest ablation of the atrioventricular junction by transcatheter delivery of high energy unmodified DC shocks to the His bundle region for the treatment of patients with drug-refractory supraventricular arrhythmias. ${ }^{12}$ This comparatively crude approach committed the patient to a permanent pacemaker and was associated with significant risk of barotrauma, but nevertheless its advent marked the beginning of a new era of interventional electrophysiology. Since 1982 improvements in catheter design, power sources, and mapping techniques have transformed catheter ablation from an investigational procedure into an indispensable therapeutic tool that has supplanted surgery and antitachycardia pacing in the management of the Wolff-Parkinson-White syndrome and junctional tachycardias and is also being applied with increasing success to various other arrhythmias including atrial flutter, ectopic atrial tachycardia, and some cases of ventricular tachycardia.

\section{Technological developments: power sources and catheters}

The original approach to catheter ablation used high-energy DC shocks of 150-400 J from a conventional defibrillator. ${ }^{12}$ These discharges generate high voltages at the catheter tip (the primary determinant of local tissue damage) but may also cause electrical arcing and barotrauma, with the risk of cardiac rupture, coronary artery spasm, diffuse myocardial damage, and ventricular arrhythmias. ${ }^{3}$ In practice, the unacceptable incidence of procedure related complications and deaths associated with high-energy DC ablation has stimulated research into alternative, safer modes of energy delivery for catheter ablation. To date, only two other power sources have been fully developed and evaluated in clinical studies. Low energy DC ablation is achieved with brief time-constant capacitive discharges, enabling delivery of high voltages with minimal or no arcing even within confined spaces such as the coronary sinus. ${ }^{45}$ The use of a custom-built elliptical electrode further increases the arcing threshold. ${ }^{4}$ This system has an excellent safety and efficacy record but it requires general anaesthesia and results in a relatively high incidence of stunning with early recurrence (for example, of accessory pathway conduction). ${ }^{6}$ The most widely used power source for catheter ablation at present is radiofrequency current. ${ }^{78}$ Systems designed for intracardiac use deliver continuous, unmodulated sine wave outputs in the range $0 \cdot 1-1 \cdot 5$ $\mathrm{M} \mathrm{Hz}$ which cause resistive heating and coagulative necrosis of myocardial tissue around the electrode tip once the temperature at the interface reaches approximately $48^{\circ} \mathrm{C}$. Power density falls off exponentially as a function of distance from the electrode tip and the process of tissue heating reaches a steady state within the first 10-40 seconds of current application, after which there is no further lesion expansion. These biophysical characteristics allow controlled delivery of discrete lesions with a high degree of safety and precision, adequate even for demanding tasks such as selective atrioventricular nodal modification. Furthermore, the lack of neuromuscular stimulation at high frequencies obviates the need for general anaesthesia. On the other hand, lesion generation is critically dependent on excellent electrode-tissue contact, which may be difficult to achieve, and current delivery is not infrequently interrupted by abrupt rises in system impedance caused by coagulum formation around the electrode tip, necessitating complete removal and cleansing of the catheter. ${ }^{78}$ Because of the small size and shallow depth of the lesions generated, radiofrequency ablation requires extremely precise mapping and may be inadequate for dealing with substrates such as subendocardial ventricular tachycardia foci caused by previous myocardial infarction. In view of
Correspondence to: Dr Y Bashir, Department of Cardiology, John Radcliffe Hospital, Headingto
Oxford OX3 9DU. 
these drawbacks, there is continuing interest in the development of new power sources such as microwave energy that may permit controlled delivery of larger myocardial lesions than radiofrequency current without the same dependence on optimal electrodetissue contact. ${ }^{9}$

There have also been important advances in the design of ablation catheters. Of particular significance has been the introduction of catheters with deflectable tips to facilitate steering and positioning along the mitral/tricuspid annulus. These catheters are now available with a range of different curves to accommodate variations in cardiac anatomy. The use of large $4 \mathrm{~mm}$ distal electrodes for delivery of radiofrequency current in place of the original $2 \mathrm{~mm}$ tips has been shown to maximise lesion size in vitro ${ }^{8}$ and to improve overall success rates in clinical practice. ${ }^{10}$ Finally, with the recognition that temperature at the tissue-electrode interface is a key determinant of lesion size and coagulum formation, there has been interest in the use of catheters equipped with tip thermistors for continuous temperature monitoring and feedback adjustment of power output to optimise tissue heating while reducing the chance of a rise in impedance. ${ }^{11}$ The clinical usefulness of this technology has not yet been established.

\section{Atrioventricular nodal ablation}

Catheter ablation of the atrioventricular junction with implantation of a permanent pacemaker is no longer regarded as an appropriate therapy for junctional tachycardias but has an important role in the management of patients with drug-refractory paroxysmal or chronic atrial fibrillation, provided that their symptoms can be attributed to rapid ventricular rates, rather than just to loss of atrial transport. Atrioventricular nodal ablation may also be indicated in some cases of atrial flutter and atrial tachycardia when attempts at curative ablation of the arrhythmia substrate have failed (see below). The original technique of atrioventricular nodal ablation with DC shocks was successful in approximately $70-80 \%$ of cases but has been largely supplanted by the use of radiofrequency current. Despite its impeccable safety record, early experience with radiofrequency catheter ablation was disappointing because complete atrioventricular block could only be produced in $50-60 \%$ of cases. ${ }^{12} 13$ However, with the introduction of $4 \mathrm{~mm}$ tip ablation catheters, the success rate has increased to around $90 \%$ with a reduction in the number of current applications required per case. ${ }^{1014}$ The standard technique involves positioning the catheter on the tricuspid annulus, slightly proximal and inferior to the site of maximal His deflection and avoiding the central fibrous body. No electrogram pattern seems to be universally effective, though most investigators suggest that a large atrial potential with a small His deflection is optimal. Current application at successful sites frequently produces transient junctional tachycardias before the onset of complete atrioventricular block. In the small proportion of patients (approximately 10\%) where this fails, a highly effective alternative approach is to introduce the ablating catheter retrogradely into the left ventricle and deliver lesions from the mitral annulus at sites where the maximum His potential can be recorded. In two recent series, the use of this technique in difficult cases has boosted the overall success rate with radiofrequency energy to close to $100 \% .{ }^{1516}$ Resumption of atrioventricular conduction after the first 30-45 minutes occurs in less than $10 \%$ of cases, and repeat procedures do not seem to be associated with any additional problems. Low energy DC shocks produce complete heart block in approximately $90 \%$ of cases and should be considered if radiofrequency ablation by both right and left sided approaches proves ineffective. ${ }^{17}$

\section{Accessory atrioventricular pathways}

Accessory pathways represent ideal targets for curative ablation because they generally occur in the absence of structural heart disease and can be selectively destroyed without altering normal atrioventricular conduction or cardiac function. Surgical division has been successfully used as a definitive therapy for more than 20 years in patients with drug-refractory atrioventricular reentrant tachycardias or rapid pre-excited atrial fibrillation. The earliest transcatheter approach involved delivery of high energy DC shocks in the vicinity of the coronary sinus os for ablation of posteroseptal bypass tracts and achieved a modest success rate of around $60-70 \% .^{18}$ Subsequently, Warin et al successfully ablated over $90 \%$ of 254 pathways in all locations with DC shocks using a direct endocardial approach with scrupulous attention to mapping, including recording and verification of accessory pathway potentials. ${ }^{19}$ After Borggrefe et al's initial description of catheter ablation of an accessory pathway with radiofrequency energy in $1987,{ }^{20}$ several groups have now published their experience with this technique in large series of patients with both pre-excited and concealed bypass tracts at all locations. ${ }^{21-25}$ Excellent results were consistently reported with success rates of $90 \%$ or better, no deaths, and an extremely low $(2-4 \%)$ incidence of serious complications; these included rare cases of pericarditis/tamponade, inadvertent atrioventricular block during ablation of anteroseptal pathways, cerebral emboli, and vascular access problems. In the Multicentre European Radiofrequency Survey of 2211 patients undergoing pathway ablation, the incidence of serious complications was also around $3.5 \%$, but there were three deaths $(0 \cdot 14 \%)$ (G Hindricks, personal communication). Accessory pathway conduction resumes after up to $10 \%$ of successful ablations, ${ }^{26}$ but the procedure can be repeated with equal efficacy and safety, and there have been no reports of recurrences after a second 
successful ablation. This remarkably favourable experience has rendered surgical division of accessory pathways virtually obsolescent, and has been a major factor in the recent emergence and growth of catheter ablation as a clinical discipline.

The high success rates that are now being routinely achieved for ablation of accessory pathways are primarily related to improvements in catheter technology and mapping techniques rather than specifically to the use of radiofrequency current as the power source. Indeed, similar results have been obtained with both high energy ${ }^{19}$ and low energy DC shocks. ${ }^{6}$ Two different approaches have been used for pathway localisation. Jackman et al advocated delivery of lesions only at sites where accessory pathway potentials can be recorded and distinguished from local atrial and ventricular activity by programmed stimulation techniques. ${ }^{21}$ Their approach is intellectually appealing and highly effective $(99 \%$ success rate with a median of three lesions delivered per case) but is very time-consuming, with procedure durations averaging more than 8 hours. Many appropriate target sites are rejected because the accessory pathway signal cannot be distinguished from the local atrial or ventricular electrogram. Because of these limitations, most groups have adopted a more pragmatic approach. ${ }^{23-25}$ Lesions are delivered at sites where the atrioventricular interval is short and local ventricular activation is early relative to the onset of the QRS complex on the surface ECG, and no attempt is made to verify possible pathway potentials. Although this method is less precise (typically a median of 8-10 lesions are required per case to achieve a $90 \%$ success rate), it has the crucial advantage of shortening average procedure duration to around $2 \cdot 5-4$ hours.

The major limitation of radiofrequency catheter ablation at present is the highly variable procedure duration. ${ }^{21-25}$ Although ablation of most pathways is straightforward, average procedure times remain long owing to a substantial minority of difficult cases. The associated increase in the exposure of patients and operators to radiation is a further drawback. ${ }^{27}$ With the role of the technique itself now firmly established in clinical practice, more attention is being paid to identifying and solving the methodological problems that cause difficulty in individual cases. Catheter manipulation and stabilisation of the tip on the atrioventricular annulus are potential sources of trouble. Good stability is particularly hard to obtain when the catheter is positioned along the tricuspid annulus for ablation of right sided pathways, and this often results in transient interruption of pathway conduction. Possible solutions include the use of dumb-bell electrode tips and braided tip catheters, use of a subclavian rather than a femoral approach, or prolapsing the catheter into the right ventricle with the tip retroflexed back onto the annulus to provide additional support. With left sided pathways, stability is usually less of a problem when the mitral annulus is approached through the standard retrograde aortic route. However, manipulation may be extremely awkward in a few cases, particularly in older patients with a tortuous aorta, and in this situation a transseptal approach to the mitral annulus has proved a highly effective alternative. ${ }^{28}$ Posteroseptal pathways account for the highest proportion of difficult cases and failures in most series. In this anatomically complex region, there is the additional problem of accurate localisation: bypass tracts can be inserted at various sites on the tricuspid or mitral annuli or along the course of the coronary sinus and may traverse the posteroseptal space obliquely to discordant atrial sites. ${ }^{29}$ It is not unusual to attempt both right and left sided approaches to the septal region in an individual patient before success is obtained. Where standard methods have failed, ablation has been achieved by use of a double-electrode configuration with bipolar current delivery between sites along the tricuspid annulus and mitral annulus ${ }^{30}$; the mechanism is unknown but could involve interruption of bypass tracts deep within the posteroseptal space.

Other measures that have been adopted in an attempt to streamline catheter ablation procedures include dispensing with a diagnostic study in cases of manifest preexcitation and the use of a single catheter technique for mapping/ablation. ${ }^{3132}$ Because total procedure duration closely parallels the number of attempted ablation sites per case, there has also been interest in maximising the efficiency of mapping techniques used for pathway localisation. Several groups have analysed local electrogram characteristics at successful and failed sites in an attempt to identify optimum mapping criteria, ${ }^{32-34}$ but have reached markedly different conclusions, probably owing to variations in data selection and methods. For example, Calkins et al suggested that sites exhibiting stable electrograms, early local ventricular activation, and a possible accessory pathway potential would be associated with a $57 \%$ probability of success, ${ }^{33}$ but this figure was achieved by selective analysis of all the successful sites and only a proportion of the unsuccessful sites. In studies that analysed all attempted ablation sites, the same electrogram criteria yielded positive predictive accuracies of less than $25 \%{ }^{32}$ In general, mapping criteria based on local electrogram characteristics (other than verified accessory pathway potentials) have proved to be relatively non-specific for selecting appropriate target sites. This remains an important factor in the long procedure times required for some cases and reflects inherent limitations of radiofrequency technology itself, namely the need for both extremely precise positioning and excellent tissueelectrode contact to destroy such a discrete structure as an accessory pathway. Monitoring of tip temperature allows incorrect positioning and inadequate tissue heating at unsuccessful target sites to be distinguished and may lead to a limited improvement in 
efficacy ${ }^{11}$ : but a more definitive solution will probably depend on the development of alternative power sources such as microwave energy that enable controlled delivery of larger myocardial lesions without the same critical dependence on electrode-tissue contact. ${ }^{9}$

\section{Atrioventricular nodal reentrant tachycardia}

Atrioventricular nodal re-entrant tachycardia (AVNRT) is the other common form of junctional tachycardia. The critical components of the re-entrant circuit are a fast pathway (usually the retrograde limb) and a slow pathway with a longer conduction time. Though such a model is almost certainly oversimplistic, it has provided the conceptual framework for the development of ablation techniques for AVNRT. Detailed mapping at electrophysiological studies and surgery suggests that these pathways are anatomically distinct in most patients, with the fast pathway situated anteriorly close to the His bundle and the slow pathway located more posteriorly in perinodal tissue close to the coronary sinus. ${ }^{35}$ The high degree of precision attainable with radiofrequency catheter ablation has for the first time enabled selective destruction of one or other limb of the re-entrant circuit with preservation of antegrade atrioventricular conduction.

Initial work focused on ablation of fast pathway conduction. ${ }^{36}$ The technique essentially resembles that used for atrioventricular junction ablation, in that the catheter is positioned just proximal to the His bundle region and current is delivered until conduction blocks in the fast pathway as evidenced by prolongation of the PR and AH intervals. The therapeutic end points include PR prolongation and abolition or prolongation of retrograde ventriculoatrial conduction. This approach has proved successful in approximately $90 \%$ of cases and has several advantages, including simplicity, clearly defined end points, and the fact that it is not dependent on inducibility of tachycardia at baseline. The most serious drawback is the risk of inducing complete atrioventricular block, which was $8 \%$ and $21 \%$ in two series from major centres. ${ }^{36} 37$ Furthermore, the observation of high grade atrioventricular block without any alteration of the reentrant mechanism in some cases, suggests that the lower turn-around point of AVNRT may be located intranodally and may be separated from the His bundle by a common pathway that is also part of the atrioventricular node. ${ }^{38}$ Another problem is that uncommon fast-slow AVNRT may emerge in some of the cases in which retrograde ventriculoatrial conduction is modified but not abolished. ${ }^{36} 37$

Selective slow pathway ablation was first described in $1990^{39}$ and now several groups have published large series with success rates approaching $100 \%$ in both common (slow-fast) and uncommon (fast-slow) AVNRT. ${ }^{374042}$ In contrast to fast pathway modification, non-inducibility of tachycardia rather than complete abolition of slow pathway conduction has been used as the major therapeutic end point in most studies, and evidence of residual anterograde slow pathway conduction and echo beats may be demonstrable in up to $50 \%$ of successfully treated cases. ${ }^{3741} 42$ Two different techniques have been described. Jackman et al identified and delivered lesions only at sites where slow pathway activation could be detected. ${ }^{42}$ Although retrograde slow pathway conduction was demonstrable in fewer than $50 \%$ of the patients, slow pathway potentials could usually be recorded during sinus rhythm as distinct deflections falling after the atrial potential and dissociated from local atrial activity by pacing manoeuvres. Inducibility of AVNRT was abolished in $78 / 80$ cases (98\%) with a median of only two lesions delivered. ${ }^{42}$ Using a similar technique, Haissaguerre et al achieved $100 \%$ success again with a median of two lesions per case in a consecutive series of 64 patients. ${ }^{41}$ The successful sites were mostly located along the tricuspid annulus in the vicinity of the coronary sinus or more anteriorly in the mid-septal region, but in a few patients ablation was achieved from within the coronary sinus os or even more posteriorly along the tricuspid annulus. Despite these excellent results, using electrophysiological mapping to guide slow pathway modification is laborious and time-consuming, with average procedure durations of 8 hours in one study and 3-7 hours in the other. The alternative technique for slow pathway ablation involves a stepwise anatomical approach, delivering a succession of lesions along the septal leaflet of the tricuspid valve starting posteriorly, close to the coronary sinus os, and moving gradually anteriorly until inducibility of AVNRT is abolished. Adopting this approach, Kay et al were successful in $30 / 34$ consecutive cases, ${ }^{40}$ and Jazayeri et al reported similar results. ${ }^{37}$ Recording of slow pathway potentials was not necessary for success. The use of anatomical landmarks to select the ablation sites requires delivery of more lesions per case than recording slow pathway potential, but is much less time-consuming, with average procedure durations of less than 2.5 hours in two recent series. $^{3743}$ Irrespective of which technique is used the major advantage of modification of the slow pathway is that the risk of atrioventricular block seems to be extremely low though not negligible. In the Multicentre European Radiofrequency Survey (MERFS), the incidence of complete heart block was $2 \cdot 1 \%$ among 290 patients undergoing modification of the slow pathway compared with $6 \cdot 2 \%$ among 240 patients undergoing modification of the fast pathway. ${ }^{44}$ It is now recognised that there can be considerable anatomical overlap between fast and slow pathway ablation sites in some patients, and inadvertent destruction of the fast pathway or complete interruption of atrioventricular nodal conduction may occasionally result from attempted modification of the slow 
pathway, even when lesions are delivered well posterior to the His bundle region in the midtriangle of Koch. ${ }^{4042}$ For this reason it is unlikely that the small risk of provoking complete heart block could be totally eliminated.

Recently, Langberg et al prospectively compared the anterior (fast pathway) and posterior (slow pathway) approaches to ablation of AVNRT in a randomised study of 50 consecutive patients with crossover if the initial approach proved unsuccessful after 1 hour or application of 10 lesions. ${ }^{45}$ The primary success rates of the two techniques were similar (55\% $v 68 \%)$ and, of particular note, all patients in whom the initial approach failed were successfully treated by the alternative technique without complete heart block developing. These results do not support theoretical concerns that attempts at ablation of the fast pathway after failure of ablation of the slow pathway might result in an increased risk of high-grade atrioventricular block, though caution is still advised in this situation.

In summary, slow pathway ablation is now widely regarded as the best technique because of its high success rate and low probability of causing complete acute heart block. However, because the risk of atrioventricular block cannot be totally eliminated and little is as yet known about long-term effects on the integrity of the conduction system, the procedure should probably only be offered to patients with troublesome symptoms that cannot be controlled medically. Fast pathway ablation may have a limited role for treating patients in whom attempts at destroying the slow pathway have failed and in those in whom tachycardia is not reliably inducible at baseline so that slow pathway modification is not technically feasible.

\section{Other arrhythmias}

Atrial flutter is less common than atrial fibrillation but cannot be adequately controlled by medical therapy in many cases. Recent studies have shown that the common form or type 1 atrial flutter is caused by a reentrant circuit in the right atrium, incorporating a zone of slow conduction that is located in the low posteroseptal region. There are reports of successful ablation of atrial flutter in a few patients by delivery of either high or lowenergy DC shocks in this zone. ${ }^{464}$ An anatomically based approach seems to be as likely to work as a map-guided one. Recently, Feld et al reported impressive results with radiofrequency current in a series of 12 patients with atrial flutter. ${ }^{48}$ Both activation and entrainment mapping were performed and the zone of slow conduction was identified either posterior or inferior to the os of the coronary sinus. Delivery of lesions at these sites resulted in abolition of atrial flutter in $10 / 12$ patients with only two recurrences (both within the first month). Calkin's et al obtained similar results. ${ }^{49}$ Though there are few data on long-term outcome these prelimi- nary reports suggest that the role of radiofrequency ablation in the management of atrial flutter may be increasing.

Recently, three groups reported $>90 \%$ success rates with radiofrequency ablation of ectopic atrial tachycardia at both left and right atrial foci. ${ }^{50-52}$ Though this is a relatively uncommon form of supraventricular tachycardia, a significant proportion of the cases present as incessant arrhythmias in children or young adults, and may eventually lead to dilated cardiomyopathy and heart failure. Accordingly, intensive therapy may be justified to prevent or reverse the haemodynamic consequences of uncontrolled chronic tachycardia.

The role of catheter ablation techniques in ventricular tachycardia (VT) is less well established. The most suitable patients are those with incessant or virtually incessant, haemodynamically stable VT of a single configuration. Success rates of $50-70 \%$ were achieved with direct current ablation combined with detailed endocardial mapping of the tachycardia exit site and/or the zone of slow conduction. ${ }^{353}$ There are only limited data on the use of radiofrequency ablation in this setting. Borggrefe et al reported a $61 \%$ success rate in 23 patients with refractory ventricular tachycardia (16 with underlying coronary artery disease), but in five cases direct current ablation was needed as well. ${ }^{54}$ More encouragingly, Morady et al successfully ablated 16/20 arrhythmia configurations $(80 \%)$ in 15 patients with haemodynamically stable uniform VT using radiofrequency energy alone. ${ }^{55}$ However, the study population was carefully selected and represented fewer than $10 \%$ of patients with coronary artery disease referred to that centre for management of symptomatic ventricular arrhythmias. The lesions generated with radiofrequency current may be too small or superficial to destroy some subendocardial arrhythmic foci secondary to prior myocardial infarction. By contrast, success rates close to $100 \%$ were recently reported for radiofrequency ablation of ventricular tachycardia in patients with structurally normal hearts ${ }^{56}$ and in patients with a macroreentrant mechanism where selective destruction of the right bundle branch was effective. ${ }^{57}$

1 Scheinman MM, Morady F, Hess DS, Gonzales R. Catheter-induced ablation of the atrioventricular junction to control refractory supraventricular arrhythmias. tion to control refractory

2 Gallagher JJ, Svenson RH, Kasell JH, et al. Catheter technique for closed-chest ablation of the atrioventricular conduction system: a therapeutic alternative for the conduction system: a therapeutic alternative for the Engl F Med 1982;306:194-200.

3 Evans Jr. GT, Scheinman MM, Zipes DP, et al. Percutaneous Cardiac Mapping and Ablation Registry final summary of results. $P A C E 1988 ; 11: 1621-4$.

4 Ahsan AJ, Cunningham D, Rowland E, Rickards AF Catheter ablation without fulguration: Design and performance of a new system. PACE 1989;12:131-5.

5 Lemery R, Leung TK, Levallee E, et al. In vitro and in vivo effects within the coronary sinus of nonarcing shocks using a new system of low energy DC ablation. Circulation 1991;83:279-93.

6 Lemery R, Talajic M, Roy D, et al. Success, safety, and late electrophysiological outcome of low-energy directcurrent ablation of patients with the Wolff-ParkinsonWhite syndrome. Circulation 1992;85:957-62.

7 Huang SKS. Advances in applications of radiofrequency 
current to catheter ablation therapy. PACE 1991;14: $28-42$.

8 Kalbfleisch SJ, Langberg JJ. Catheter ablation with radiofrequency energy: Biophysical aspects and clinical applications. $\mathcal{F}$ Cardiovasc Electrophysiol 1992;3:173-86.

9 Cohen TJ, Coggins D, Chin MC, Stauffer P, Scheinman MM, Langberg J. Microwave ablation of ventricular myocardium: effects of varying duration on lesion

10 Jackman WM, Wang X, Friday KJ, et al. Catheter ablation of the atrioventricular junction using radiofrequency current in 17 patients. Comparison of standard and large tip catheter electrodes. Circulation 1991;83: $1562-76$

11 Langberg J, Calkins H, El-Atassi R, et al. Temperature monitoring during radiofrequency catheter ablation of monitoring during radiofrequency catheter ablation

12 Langberg J, Chin MC, Rosenquist $M$, et al. Catheter ablation of the atrioventricular junction with radiofrequency energy. Circulation 1989;80:1527-35.

13 Evans Jr. GT, Huang WH and the CAR Investigators. Comparison of direct current and radiofrequency energy for catheter ablation of the atrioventricular junction: results of a prospective multicenter study [abstr]. Circulation 1990;80(Suppl II):II-323.

14 Yeung-Lai-Wah JA, Alison JF, Lonergan L, Mohama R, Leather R, Kerr CR. High success rate of atroventricular node ablation with radiofrequency energy. $7 \mathrm{Am}$ Coll Cardiol 1991;18:1753-8.

15 Sousa, J, El-Atassi R, Rosenheck S, Calkins H, Langberg J, Morady F. Radiofrequency catheter ablation of the atrioventricular junction from the left ventricle. Circulation 1991;84:567-71.

16 Trohman RG, Simmons TW, Moore SL, Firstenberg MS, Williams D, Maloney JD. Catheter ablation of the atrioventricular junction using radiofrequency energy atrioventricular junction using radiofrequency energy and a bilater

17 Rowland E, Cunningham D, Ahsan A, Rickards AF. Transvenous ablation of atrioventricular conduction with a low energy power source. Br Heart $\mathcal{f} 1989 ; 62$ : 361-6.

18 Morady F, Scheinman MM, Khou WH, et al. Long-term results of catheter ablation of a posteroseptal accessory atrioventricular connection in 48 patients. Circulation 1989;79:1160-70.

19 Warin JF, Haissaguerre M, D'Ivernois C, Le Metayer P, Montserrat $P$. Catheter ablation of accessory pathways: technique and results in 248 patients. PACE 1990; 13:1609-14.

20 Borggrefe M, Budde T, Podczeck A, Breithardt G. High frequency alternating current ablation of an accessory pathway in humans. $₹ \mathrm{Am}$ Coll Cardiol 1987;10:576-82.

21 Jackman WM, Wang X, Friday KJ, et al. Catheter ablation of accessory atrioventricular pathways (WolffParkinson-White syndrome) by radic

22 Calkins $\mathrm{H}$, Sousa J, El-Atassi R, et al. Diagnosis and cure of the Wolff-Parkinson-White syndrome or paroxysmal supraventricular tachycardias during a single electrophysiologic test. N Engl f Med 1991;324:1612-8.

23 Schluter M, Geiger M, Siebels J, Duckeck, Kuck KH Catheter ablation using radiofrequency current to cure symptomatic patients with tachyarrhythmias related to an accessory atrioventricular pathway. Circulation 1991;84:1644-61.

24 Lesh MD, van Hare GF, Schamp DJ, et al. Curative percutaneous catheter ablation using radiofrequency energy for accessory pathways in all locations: results in 100 consecutive patients. F Am Coll Cardiol 1992;19:1303-9.

25 Calkins H, Langberg JJ, Sousa J, et al. Radiofrequency catheter ablation of accessory atrioventricular conneccatheter ablation of accessory atrioventricular conn in 250 patients. Circulation 1992;85:1337-46.

26 Langberg J, Calkins H, Kim N-Y, et al. Recurrence of conduction in accessory atrioventricular connections after initially successful radiofrequency catheter abla tion. 7 Am Coll Cardiol 1992;19:1588-92.

27 Calkins H, Niklason L, Sousa J, El-Atassi R, Langberg JJ, Morady F. Radiation exposure during radiofrequency catheter ablation of accessory atrioventricular connections. Circulation 1991;84:2376-82.

28 Natale A, Klein GJ, Yee R, Wathen M, Wolfe K. Different approaches for radiofrequency ablation of left lateral accessory pathways. Eur Heart $\mathcal{f}$ 1992;13 (suppl.):2.

29 Wang X, Jackman WM, McClelland J, et al. Sites of successful radiofrequency ablation of posteroseptal accessory pathways. [Abstr]. PACE 1992;15:535.

30 Bashir Y, Heald SC, O'Nunain S, Camm AJ, Ward DE. Radiofrequency ablation of posteroseptal pathways using a tricuspid annulus-mitral annulus electrode configuration. $₹$ Am Coll Cardiol 1993;22:550-6.

31 Kuck KH, Schluter M. Single-catheter approach to radiofrequency current ablation of left-sided accessory pathways in patients with Wolff-Parkinson-White synpathways in patients with Wolff-Park
drome. Circulation 1991;84:2366-75.

32 Bashir Y, Heald SC, Katritsis D, Hamouda M, Camm AJ, Ward DE. Radiofrequency ablation of accessory atrioventricular pathways: predictive value of local electro- gram characteristics for identification of successful target sites. Br Heart f 1993;69:315-21.

33 Calkins H, Kim Y-N, Schmaltz S, et al. Electrogram criteria for identification of appropriate target sites for radiofrequency catheter ablation of accessory atrioventricular connections. Circulation 1992;85:565-73.

34 Silka MJ, Kron J, Halperin BD, et al. Analysis of local electrogram characteristics correlated with successful radiofrequency catheter ablation of accessory atrioventricular pathways. PACE 1992;15:1000-7.

35 Sung RJ, Waxman HL, Saksena S, Juma Z. Sequence of retrograde atrial activation in patients with dual atrioventricular nodal pathways. Circulation 1981;64: 1059-67.

36 Lee MA, Morady F, Kadish A, et al. Catheter modification of the atrioventricular junction with radiofrequency energy for control of atrioventricular nodal reentry tachycardia. Circulation 1991;83:827-35.

37 Jazayeri MR, Hempe SL, Sra JS, et al. Selective transcatheter ablation of the fast and slow pathways using radiofrequency energy in patients with atrioventricular nodal reentrant tachycardia. Circulation 1992;85 1318-28.

38 Deshpande S, Blanck Z, Dhala A, et al. Lower 'turn around' structure in atrioventricular nodal reentran tachycardia: intranodal or extranodal? [abstr] $\mathcal{f} \mathrm{Am} \mathrm{Coll}$ Cardiol 1992;19:144A.

39 Roman CA, Wang X, Friday KJ, et al. Catheter technique for selective ablation of the slow pathway in AV nodal reentrant tachycardia [abstr]. PACE 1990;13:498.

40 Kay GN, Epstein AE, Dailey SD, Plumb VJ. Selective radiofrequency ablation of the slow pathway for the radiofrequency ablation of the slow pathway for the
treatment of atrioventricular nodal reentrant tachycartreatment of atrioventricular nodal
dia. Circulation 1992;85:1675-88.

41 Haissaguerre M, Gaita F, Fischer B, et al. Elimination of atrioventricular nodal reentrant tachycardia using discrete slow potentials to guide application of radiofrequency energy. Circulation 1992;85:2162-75.

42 Jackman WM, Beckman $\mathrm{KJ}$, McClelland $\mathrm{JH}$, et al. Treatment of supraventricular tachycardia due to atrioventricular nodal reentry by radiofrequency catheter ablation of slow pathway conduction. $N$ Engl $f \mathrm{Med}$ 1992;327:313-8

43 Wu D, Yeh S-J, Wang CC, Wen M-S, Lin F-C. A simple technique for selective radiofrequency ablation of the slow pathway in atrioventricular node reentrant tachycardia. $\mathcal{F}$ Am Coll Cardiol 1993;21:1612-21.

44 Hindricks $G$, Haverkamp W, Borggrefe $M$. Incidence of complete atrioventricular block in 808 patients after radiofrequency modification of the atrioventricular node [abstr]. Eur Heart $\mathcal{f} 1993 ; 14$ (suppl):257.

45 Langberg J, Leon A, Borganelli M, et al. A randomized, prospective comparison of anterior and posterior prospective comparison of anterior and posterior
approaches to radiofrequency catheter ablation of atrioapproaches to radiofrequency catheter ablation of atrio-
ventricular nodal reentrant tachycardia. Circulation ventricular nodal

46 Saoudi N, Atallah G, Kirkorian G, Touboul P. Catheter ablation of the atrial myocardium in type 1 atrial flutter. Circulation 1990;81:762-71

47 O’Núnáin S, Linker NJ, Sneddon JF, Debbas NMG, Camm AJ, Ward DE. Catheter ablation by low energy DC shocks for successful management of atrial flutter. Br Heart $\mathcal{F}$ 1992;67:67-71

48 Feld GK, Fleck RP, Chen PS, et al. Radiofrequency catheter ablation for the treatment of human type atrial flutter. Circulation 1992;86:1233-40.

49 Calkins H, El-Atassi R, Kalbfleisch SJ, Langberg JJ, Morady F. Catheter ablation of atrial flutter using radiofrequency energy. Circulation 1992;86(suppl. 1): I-723.

50 Walsh EP, Saul JP, Hulse JE, et al. Transcatheter ablation of ectopic atrial tachycardia in young patients using
radiofrequency current. Circulation 1992;86:1138-46.

51 Tracy CM, Swartz JF, Fletcher RD, et al. Radiofrequency catheter ablation of ectopic atrial tachycardia using paced activation sequence mapping. $\mathcal{f} \mathrm{Am}$ Coll Cardiol 1993;21:910-7.

52 Kay GN, Chong F, Epstein AE, Dailey SM, Plumb VJ. Radiofrequency ablation for treatment of primary atrial tachycardias. f Am Coll Cardiol 1993;21:901-9.

53 Morady F, Kadish A, Rosenheck S, et al. Concealed entrainment as a guide for catheter ablation of ventricular tachycardia in patients with prior myocardial infarction. F Am Coll Cardiol 1991;17:678-89.

54 Borggrefe $\mathrm{M}$, Willems S, Chen X, et al. Catheter ablation of ventricular tachycardia using radiofrequency current. Herz 1992;17:171-8.

55 Morady F, Harvey M, Kalbfleisch SJ, El-Atassi R, Calkins $\mathrm{H}$, Langberg JJ. Radiofrequency catheter ablation of ventricular tachycardia in patients with coronary artery disease. Circulation 1993;87:363-72.

56 Klein LS, Shih H-T, Hackett K, Zipes DP, Miles WM. Radiofrequency catheter ablation of ventricular tachycardia in patients without structural heart disease. Circulation 1992;85: 1666-74.

57 Cohen TJ, Chien WW, Lurie KG, et al. Radiofrequency catheter ablation for treatment of bundle branch catheter ablation for treatment of bundle branch follow-up. $\mathcal{f} \mathrm{Am}$ Coll Cardiol 1991;18:1767-73. 